Document downloaded from:

http://hdl.handle.net/10251/65388

This paper must be cited as:

Giner Sanz, JJ.; Ortega Navarro, EM.; Pérez-Herranz, V. (2015). Optimization of the electrochemical impedance spectroscopy measurement parameters for PEM fuel cell spectrum determination. Electrochimica Acta. 174:1290-1298. doi:10.1016/j.electacta.2015.06.106.

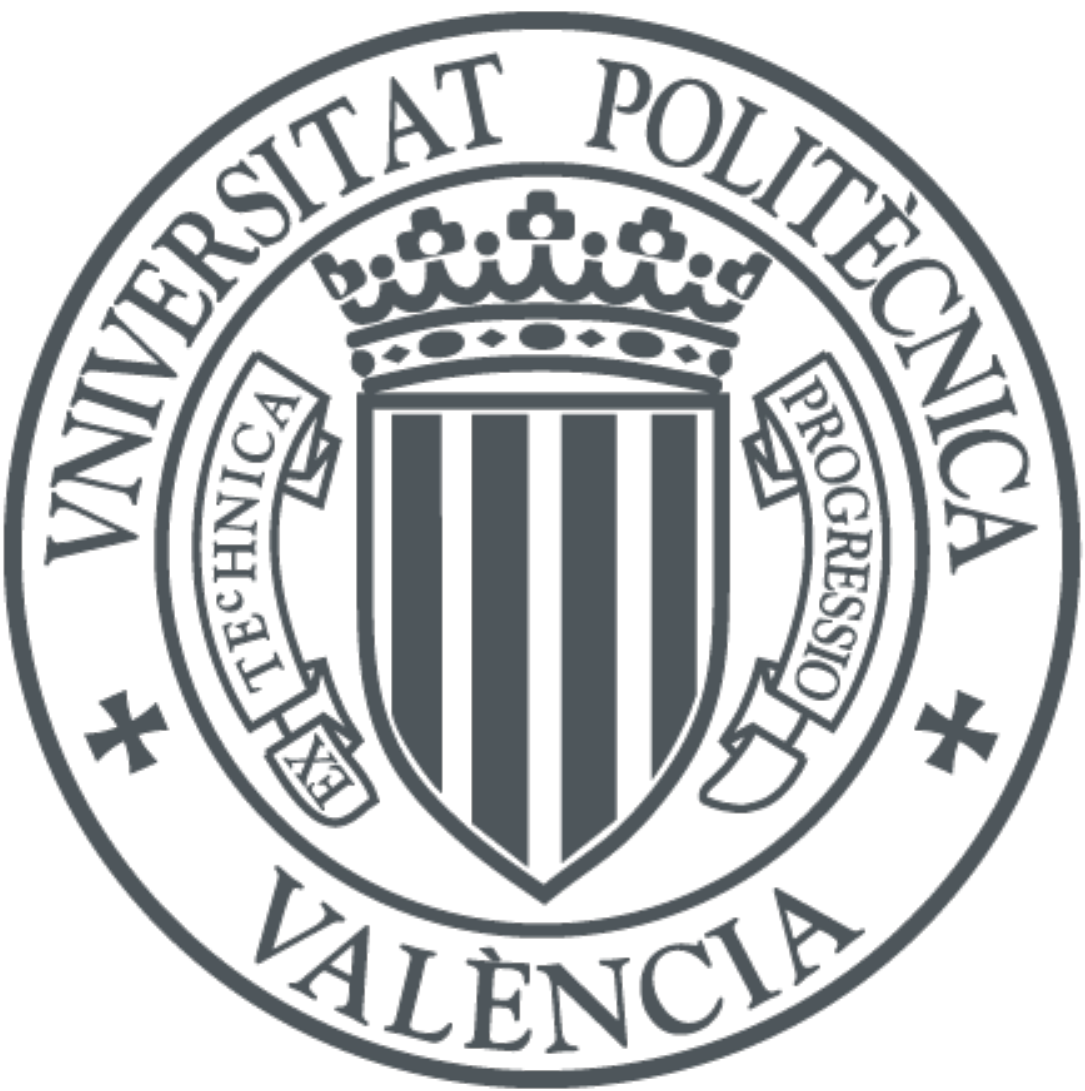

The final publication is available at

http://dx.doi.org/10.1016/j.electacta.2015.06.106

Copyright Elsevier

Additional Information 


\title{
Optimization of the electrochemical impedance spectroscopy measurement parameters for PEM fuel cell spectrum determination
}

\author{
J. J. Giner-Sanz, E. M. Ortega, V. Pérez-Herranz* \\ IEC group, Depto. Ingeniería Química y Nuclear, Universitat Politècnica de València \\ Camino de Vera S/N, 46022 Valencia, Spain \\ *Corresponding author. Tel.: +34-96-3877632; fax: +34-96-3877639; \\ E-mail address: vperez@iqn.upv.es (V.Pérez-Herranz)
}

Keywords: Electrochemical Impedance Spectroscopy; measurement parameters; optimization; factorial experimental design; PEMFC.

\section{Abstract}

Currently, electrochemical Impedance Spectroscopy (EIS) is a widely used tool for the study of electrochemical systems, in general; and fuel cells, in particular. A great effort is typically invested in the analysis of the obtained spectra; whereas, little time is usually spent optimizing the measurement parameters used to obtain these spectra. In general, the default settings provided by the control software used to perform the measurements, or the parameters used in similar systems available in literature, are selected to carry out the measurements. The goal of this work is to determine the optimal measurement parameters for obtaining impedance spectra of a commercial PEM fuel cell. In order to achieve this, a $2^{5}$ factorial design was considered. Five factors were considered, the five impedance spectroscopy measurement parameters: maximum integration time; minimum number of integration cycles; number of stabilization cycles; maximum stabilization time; and minimum cycle fraction. For each factor combination envisaged in the experimental design, the cell spectrum was obtained in given operation conditions, for which the reference spectrum of the system was known, since it had been determined in previous works. The experimentally obtained spectra were fitted to the reference electric equivalent circuit. The mean square error between the experimental data fitting and the reference spectrum fitting was determined in each case, and was used as the dependant variable for the experimental design analysis. An analysis of the variance was performed in order to determine which measurement parameters have a significant effect on the dependant variable; and a model relating the dependant variable and the measurement parameters was built. This model was used in order to obtain the optimal value of each one of the measurement parameters that minimized the mean square error of the fit obtained from the experimental data with respect to the reference fit. 


\section{Introduction}

In current days, electrochemical impedance spectroscopy (EIS) has gained significant relevance in the fuel cells field, since this electrochemical measurement technique allows obtaining information on the fuel cell internal state and on its electrochemical behaviour [1, 2]. This technique provides detailed information on the conductivity of the membrane, on the electrochemical electrode processes and on the intrinsic losses of the system [3-5]. All these data are crucial in order to tackle some of the most challenging actual issues of fuel cells, such as membrane drying and gas diffusion layer flooding $[6,7]$. For this reason, EIS has widely been applied for membrane electrode assembly optimization [8-13]; operation conditions optimization [14, 15]; degradation studies [16, 17]; control [18] and diagnosis [19-20]. The technique has been applied both, to fuel cell single cells [20-24] and to fuel cell stacks [19, 25].

The measurement of electrochemical impedance spectra consists in a frequency scan [26]: for each selected frequency an impedance measurement is done by sampling the current and voltage AC signals [27]. Usually the measurement for each frequency is performed in two steps: a stabilization step followed by an acquisition step [28]. Therefore, the time required for the measurement of the impedance for a given frequency $\left(t_{m}\right)$ is given by:

$$
t_{m}(f)=t_{s}(f)+t_{a}(f)
$$

Where $t_{m}(f)$ is the measurement time for frequency $f ; t_{s}(f)$ and $t_{a}(f)$ denote the stabilization time and acquisition time for frequency $f$, respectively.

The total duration of the measurement of the whole spectrum $\left(t_{t o t}\right)$ is given by the sum of the measurement times for all frequencies for which the impedance is measured:

$$
t_{\text {tot }}=\sum_{i=1}^{N_{f}} t_{m}\left(f_{i}\right)
$$

Where $N_{f}$ is the number of frequencies for which the impedance is measured: the number of points of the impedance spectrum. 
On the one hand, the acquisition step is defined by two parameters: the maximum integration time $\left(\tau_{I}\right)$ and the minimum number of integration cycles $\left(v_{I}\right)$. Both parameters compete against each other; and depending on the applied frequency, the acquisition segment will be defined by one of these two parameters: for high frequencies (larger than $v_{I} \cdot \tau_{I}{ }^{-1}$ ) the maximum integration time parameter is used; whereas for low frequencies (smaller than $v_{I} \cdot \tau_{I}^{-1}$ ) the minimum number of integration cycles parameter is used [29]. Therefore the acquisition segment time is given by:

$$
t_{a}(f)= \begin{cases}\tau_{I} & \text { if } \tau_{I} \geq \frac{v_{I}}{f} \\ \frac{v_{I}}{f} & \text { if } \tau_{I}<\frac{v_{I}}{f}\end{cases}
$$

On the other hand, the stabilization step is defined by three parameters: the number of stabilization cycles $\left(v_{S S}\right)$, the maximum stabilization time $\left(\tau_{S S}\right)$ and the minimum cycle fraction $\left(\xi_{S S}\right)$. These parameters are competing one another; and depending on the applied frequency, the stabilization segment will be defined by one of these three parameters: for high frequencies (larger than $v_{S S} \cdot \tau_{S S}{ }^{-1}$ ) the number of stabilization cycles parameter is used; for low frequencies (smaller than $v_{S S} \cdot \tau_{S S}{ }^{-1}$ ) the maximum stabilization time is used; and finally, for very low frequencies (smaller than $\tau_{S S}{ }^{-1}$ ) the minimum cycle fraction is used [29]. Therefore the settling segment time is given by:

$$
t_{s}(f)= \begin{cases}\frac{v_{S S}}{f} & \text { if } \tau_{S S} \geq \frac{v_{S S}}{f} \\ \tau_{S S} & \text { if } \tau_{S S}<\frac{v_{S S}}{f} \\ \frac{\xi_{S S}}{f} & \text { if } \tau_{S S}<\frac{1}{f}\end{cases}
$$

In short, the impedance spectroscopy measurement is defined by five parameters: the maximum integration time, the minimum number of integration cycles, the number of stabilization cycles, the maximum stabilization time and the minimum cycle fraction. In general, no preliminary study is done in order to determine the optimal values of these parameters for obtaining the impedance spectrum of a given system [26]. Common practices include using the default settings provided by the control software employed to perform the measurement; or using the parameters used in similar systems available in literature [28]. However, these practices may lead to suboptimal measurement parameters: a set of measurement parameters that produce a spectrum that is not the best spectrum that could be obtained in terms of the error of the spectrum with respect to the "real" spectrum. 
The main purpose of this work is to determine the optimal measurement parameters for obtaining impedance spectra of a commercial PEM fuel cell. Furthermore, this work presents a framework for the optimization of impedance measurement parameters for other systems. In order to fulfil this goal, the impedance spectrum of an individual cell of a commercial PEM fuel cell stack was measured in given operation conditions, using different impedance measurement parameters. From previous works, the impedance spectrum of the studied cell is known with significant accuracy for the operation conditions used in this work. This spectrum was used as the reference spectrum. The experimental spectra obtained for different impedance measurement parameters were compared with the reference spectrum, using a comparison parameter that quantifies the deviation of the experimental spectra from the reference spectrum. Then, an analysis of variance (ANOVA) statistical study was performed on the comparison parameter; and a regression method was used in order to obtain a black box model relating the comparison parameter value to the measurement parameters. This black box model was used to obtain the optimum measurement parameter values. 


\section{Experimental design}

A full $2^{5}$ factorial design with centerpoint was used in this work. This kind of experimental design consists in an experimental design where 5 factors are studied at only two levels: a high level and a low level [30]. Full factorial designs involve running all the $2^{5}$ combinations of the two levels of the five factors. A centerpoint consists in an experimental point defined at the central value between the high and the low level values for each one of the considered factors. Adding centerpoints to the experimental design is useful for detecting possible curvatures in the output variables [31]. This experimental design strategy is much more efficient than the traditional "one after one" experimental strategy since it requires fewer experiments to analyse the same number of input factors and allows studying the interaction between these factors [32].

In this study, 5 factors were studied at two levels: a high level $(+1)$ and a low level $(-1)$; and at a centerpoint (level 0 ). The five selected factors were the five impedance spectroscopy measurement parameters: maximum integration time; minimum number of integration cycles; number of stabilization cycles; maximum stabilization time; and minimum cycle fraction. Each of the three levels of each factor is given in table 1. The factor levels were selected according to typical default values for each one of the measurement parameters, given as well in table 1 . These default values are the values used by most of the impedance measurement softwares, such as NOVA®, Zplot ${ }^{\circ}$ or GPES/FRA $®$.

In summary, the experimental design used in this work consists in 33 treatments. Each treatment corresponds to one of the combinations of factors that are considered in the experimental design. 


\section{Methodology}

For each one of the experimental design treatments defined in section 2, the impedance spectrum of the commercial PEM fuel cell was obtained using the experimental setup described in section 4. The operation conditions were the same for all the treatments. Therefore, the only parameters that changed from one spectrum measurement to another were the measurement parameter values, introduced in section 1 . The cell's spectrum for the selected operation conditions (stated in section 4) had been determined in previous works with significant accuracy. This known spectrum was used as reference spectrum: the experimental spectra were compared, as it will be described further in this section, with the reference spectrum.

Figure 1 shows the reference spectrum and the equivalent circuit of the PEM fuel cell in the selected operation conditions. This reference spectrum was determined in a preliminary work, by measuring 150 times the spectrum in the selected operation conditions with NOVA®'s default measurement parameters, and using statistical inference tools in order to obtain the "real" spectrum with a high level of accuracy. The power of the statistical inference tools used in this work is that they are able to identify and take away the noise by repeating the measurement a large number of times (150 times): this allows obtaining the "real" spectrum from a large number of measurements.

The experimental spectra were compared to the reference spectrum: The goal is to select the measurement parameter values that lead to an experimental spectrum as close as possible to the reference spectrum. A comparison parameter had to be selected in order to quantify the deviation of the experimental spectrum from the reference one. In general, the analysis of the impedance spectra is done through equivalent circuit fitting [28]; hence, more than comparing the spectra themselves, it is preferable to compare the fitting of the experimental spectra to the reference spectrum. Consequently, in this work, the experimental spectra were fitted to the same equivalent circuit that was used for the reference spectrum, given in figure 1. The mean square error of the fitted experimental spectrum with respect of the reference spectrum, $M S E_{f i t}$, was used as comparison parameter. This parameter is defined by the following expression:

$$
M S E_{f i t}=\frac{1}{N_{f}} \cdot \sum_{i=1}^{N_{f}}\left[Z_{f i t}^{\prime}\left(\omega_{i}\right)-Z_{r e f}^{\prime}\left(\omega_{i}\right)\right]^{2}+\left[Z_{f i t}^{\prime \prime}\left(\omega_{i}\right)-Z_{r e f}^{\prime \prime}\left(\omega_{i}\right)\right]^{2}
$$

Where $\omega_{i}$ stands for the i-th measured angular frequency; $Z_{f i t}^{\prime}$ and $Z_{f i t}^{\prime \prime}$ are the real and imaginary parts of the experimental spectrum fitting for each angular frequency; 
and finally, $Z_{r e f}^{\prime}$ and $Z^{\prime \prime}{ }_{r e f}$ are the real and imaginary parts of the reference spectrum for each angular frequency. This parameter measures the distance of the fitted experimental spectrum with respect to the reference spectrum. This was the dependant variable used in the experimental design of this work.

Each one of the experimental spectra was fitted to the reference electric equivalent circuit. The mean square error between the experimental data fitting and the reference spectrum was determined in each case. Thus, for each combination of measurement parameter values the mean square error between the experimental spectrum, obtained for that combination of measurement parameters, and the reference spectrum was determined.

A statistical analysis was performed taking the mean square error as the dependant variable. Firstly, an analysis of the variance (ANOVA) was carried out in order to determine which measurement parameters have a significant effect on the deviation of the experimental spectrum from the reference spectrum; and the sign of this effect. Secondly, a regression model was built using the response surface methodology (RSM). This black box model relates the dependant variable and the measurement parameters. Both, ANOVA and RSM, are well established statistical methods [32].

Finally, the built regression model was used in order to obtain the optimal value of each one of the measurement parameters that minimized the mean square error of the fit obtained from the experimental data with respect to the reference fit: the measurement parameter values that lead to an experimental spectrum which fitting is the closest to the reference spectrum. Figure 2 summarises the methodology used in this work. 


\section{$\underline{\text { 4. Experimental }}$}

The experimental setup is represented in figure 3. Its main element is a $300 \mathrm{~W}$ commercial FC stack, provided by HeliocentriS $\AA$, composed by 20 individual cells with an effective area of $58 \mathrm{~cm}^{2}$. The air supply is provided by a compressor and the hydrogen comes from a 200 bar high-pressure storage tank. The humidification of the gas inlets is assured by a humidification system and the fuel stack operating temperature is controlled by a refrigeration system. On the one hand, the humidification system consists in two independent bubbling humidification systems, with humidification temperature control. On the other hand, the refrigeration system consists in a heat exchanger equipped with a continuous pump and a temperature controller. The reactant gases flow rates are controlled using mass flow controllers. The reactant inlet pressures are monitored by pressure gauges and are regulated using manual valves. All the relevant system temperatures are monitored by thermocouples. The overall control was done using a control computer with a Labview $\circledast$ based control program. All the experiments were carried out in open end anode mode, with constant inlet reactant flow rates: $2.1 \mathrm{NL} \cdot \mathrm{min}^{-1}$ for the hydrogen stream and $15 \mathrm{NL} \cdot \mathrm{min}^{-1}$ for the air stream. The individual cell galvanostatic impedance spectra were obtained using an Autolab® $302 \mathrm{~N}$ potentiostat/galvanostat with FRA module, controlled using NOVA® software.

As stated in section 3, all the spectra were obtained in the same cell operation conditions: The operation temperature and the humidification temperatures were set to $70^{\circ} \mathrm{C}$, and the $\mathrm{DC}$ current was set to $1 \mathrm{~A}$. The selected frequency range extended from $5 \mathrm{kHz}$ to $250 \mathrm{mHz}$, with 50 frequencies logarithmically selected. The perturbation amplitude was set to $5 \%$ of the DC current [10].

The preconditioning of the PEMFC system can heavily influence the experimental results, since the preconditioning operating point establishes the water content of the membrane, and thus determines the membrane resistance [33]. Because of this, a 15 min preconditioning before each measurement was done in order to assure that the system remained in the same working point through all the performed experiments. The preconditioning conditions were the same operation conditions used during the experiment itself, stated previously. 


\section{Results and discussion}

\subsection{Experimental spectra}

Figures 4 and 5 show examples of the obtained experimental spectra. Figure 4 gives the obtained spectrum for treatment $(-;-;-;-;-)$ : Integration time: $0.1 \mathrm{~s}$; Number of integration cycles: 1 cycle; Number of stabilization cycles: 5 cycles; Stabilization time: $1 \mathrm{~s}$; Minimum cycle fraction: 0 . And figure 5 gives the obtained spectrum for treatment $(+;+;-;-;+)$ : Integration time: $1 \mathrm{~s}$; Number of integration cycles: 5 cycles; Number of stabilization cycles: 5 cycles; Stabilization time: $1 \mathrm{~s}$; Minimum cycle fraction: 0.5 . The points represent the obtained experimental data; the dashed line represents the fitting of the experimental data to the reference equivalent circuit; and finally, the solid line is the reference spectrum. The mean square error value for each one of the spectra is displayed on the figure itself. These values were calculated from the fit of the experimental data (dashed line) and the reference data (solid line), using equation (5).

The experimental spectra are composed of a capacitive loop and a high-frequency inductive zone. It is well known that the high-frequency inductive zone is related to the cable impedance [20]. Since the aim of this work is to study the PEM fuel cell itself and not the cable connexions, only the capacitive loop was considered in this work. Therefore, the high-frequency inductive zones were removed before performing the fittings.

It can be observed that the measurement parameters have a significant effect on the obtained experimental spectrum, and therefore on the fitting of the experimental spectrum. This highlights the need to properly select the values of the measurement parameters; and hence justifies this work.

\subsection{ANOVA analysis}

As stated in section 3, after determining the value of the fit mean square parameter for each one of the experiments, an ANOVA analysis was performed. The comparison parameter $\left(M S E_{f i t}\right)$ was selected as dependant variable; whereas the measurement parameters were selected as the independent factors of the ANOVA study. The ANOVA analysis was performed for a confidence level of 95\%. Table 2 corresponds with the obtained ANOVA table; while figure 6 represents the Pareto chart extracted from the ANOVA analysis.

The ANOVA table gives the statistical significance (quantified by the $p$-value) of all the simple and the interaction effects; while the Pareto chart gives both, the sign and the 
magnitude, of the effect of each factor and each interaction on the dependant variable [31].

It can be observed that three main factors have a statistically significant effect on the dependant parameter with a $95 \%$ confidence level: the maximum stabilization time, the number of stabilization cycles and the minimum number of integration cycles. Moreover, the interaction between the maximum stabilization time and the minimum cycle fraction; and the interaction between the maximum integration time and the minimum number of integration cycles also have statistically significant effects on the dependant parameter. So, the statistically significant interactions correspond with interactions between factors that define the same measurement step: there is a significant interaction associated to the acquisition step and another one associated to the stabilization one.

In addition, it can be seen that both, the maximum stabilization time and the minimum cycle fraction, have a positive effect on the dependant variable: higher values of these factors lead to a higher value of the mean square error, and therefore to a higher deviation from the reference spectrum. These parameters define the stabilization segment for low and very low frequencies, for which the cycle times are high, and therefore the drift of the PEM fuel cell is significant. It can be deduced that for low and very low frequencies the stabilization segment causes more drawbacks than improvements on the spectrum measurement. Meanwhile, the other three measurement parameters have a negative effect on the dependant parameter: an increase in these parameters causes a drop in the mean square error, hence to experimental data closer to the reference spectrum. It can be deduced that increasing the acquisition segment time always improves the measurement (in the studied factor range); and that the stabilization segment improves the measurement only for high frequencies.

\subsection{Regression model}

As stated in section 3, after performing the ANOVA analysis, a response surface method was applied in order to obtain a regression model that related the value of the dependant parameter $\left(M S E_{f i t}\right)$ to the measurement parameters. According to the significance results extracted from the ANOVA analysis, presented in section 5.2, it can be deduced that the model should include the five measurement parameters (since three of them have significant main effects; whereas the other two appear in significant interaction terms), and the two significant interaction terms. Therefore, the following quadratic model was proposed: 


$$
M S E_{f i t}\left(\vec{x}_{f}\right)=\frac{1}{2} \cdot \vec{x}_{f}^{T} \cdot \boldsymbol{A} \cdot \vec{x}_{f}+\vec{b} \cdot \vec{x}_{f}+c
$$

Where $\vec{x}_{f}$ denotes the vector of independent factors. It has already been explained that in this case the independent factors are the measurement parameters. Thus, the factor vector is defined by the following expression:

$$
\vec{x}_{f}^{T}=\left(\begin{array}{lllll}
\tau_{I} & v_{I} & v_{S S} & \tau_{S S} & \xi_{S S}
\end{array}\right)
$$

$\boldsymbol{A}$ is the second order effect matrix. It contains the coefficients of the second order simple effects (quadratic effects) and the coefficients of the interaction effects (second order cross effects). Since the ANOVA analysis revealed that there were only two second order significant effects, the second order effect matrix is of the following form:

$$
\boldsymbol{A}=\left(\begin{array}{ccccc}
0 & a_{\tau_{I} \cdot v_{I}} & 0 & 0 & 0 \\
a_{\tau^{I} \cdot v_{I}} & 0 & 0 & 0 & 0 \\
0 & 0 & 0 & 0 & 0 \\
0 & 0 & 0 & 0 & a_{\tau_{S S} \cdot \xi_{S S}} \\
0 & 0 & 0 & a_{\tau_{S S} \xi_{S S}} & 0
\end{array}\right)
$$

Where $a_{\tau_{I} \cdot v_{I}}$ is the coefficient associated with the acquisition step interaction; and $a_{\tau_{S S} \xi_{S S}}$ is the coefficient associated with the stabilization step.

Finally, $c$ represents the model constant; and $\vec{b}$ stands for the simple effect vector, given by:

$$
\vec{b}=\left(\begin{array}{c}
b_{\tau_{I}} \\
b_{v_{I}} \\
b_{v_{S S}} \\
b_{\tau_{S S}} \\
b_{\xi_{S S}}
\end{array}\right)
$$

The proposed black box model is defined by 8 coefficients: 5 simple effect coefficients (one for each measurement parameter), 2 interaction coefficients (one for each measurement step) and a constant coefficient. As it was stated in section 2, 33 experimental points were obtained. In order to get a significant model fitting using a response surface method, it is recommended to have at least 10 experimental points more than parameters considered in the model [32]. Therefore, in this case, there are enough experimental points to obtain a significant fitting. The values of the different 
regression model coefficients were obtained using RSM, and the obtained results are given in table 3.

The model has a determination coefficient of $83.35 \%$ : this means that $83.35 \%$ of the variability of the experimental points is explained by the model. This is a significant $R^{2}$ value: it indicates that the model achieves to adequately represent the relation of the fitting mean square and the measurement parameters.

In order to confirm that the obtained model is statistically significant, the following statistical hypothesis contrast is considered:

$$
\left\{\begin{array}{rr}
H_{0}: & \text { The model is not meaningful } \\
H_{1}: & \text { The model is meaningful }
\end{array}\right.
$$

Table 4 gives the ANOVA table obtained for the above hypothesis contrast test. Since the obtained p-value is 0.0104 , it can be concluded that the model is statistically significant with a confidence level of nearly $99 \%$.

In brief, the obtained regression model is statistically meaningful; and manages to properly model the effect of the measurement parameters on the dependant parameter, $M S E_{\text {fit }}$. Therefore, the model can be accepted and considered as valid for the purposes of this study.

The validity of regression models is restrained to the domain of the data from which they are obtained from [31]. Consequently, the regression model obtained in this section is only applicable for the range of measurement parameters considered in the experimental design, and given in table 1 . A low bond vector and a high bond vector are defined:

$$
\begin{gathered}
\vec{x}_{\text {min }}=\left(\begin{array}{c}
0.1 \\
1 \\
5 \\
1.0 \\
0.00
\end{array}\right) \\
\vec{x}_{\max }=\left(\begin{array}{c}
1.0 \\
5 \\
15 \\
40.0 \\
0.50
\end{array}\right)
\end{gathered}
$$

The regression model can be written as: 


$$
\begin{array}{r}
M S E_{f i t}:\left\{\vec{x} \in \mathbb{R}^{5} \mid x_{\min _{i}} \leq x_{i} \leq\right. \\
\left.x_{\max _{i}} 0 \forall i \epsilon\{1 ; 2 ; \ldots ; 5\}\right\} \rightarrow \mathbb{R}^{+} \\
\vec{x}_{f} \rightarrow \frac{1}{2} \cdot \vec{x}_{f}{ }^{T} \cdot \boldsymbol{A} \cdot \vec{x}_{f}+\vec{b} \cdot \vec{x}_{f}+c
\end{array}
$$

\subsection{Optimization}

The goal of this study is to obtain the optimum measurement parameters that lead to an experimental spectrum as close as possible to the reference one. Therefore, the optimal measurement parameters are the parameters that minimize the mean square error, $M S E_{f i t}$. This corresponds to the following optimization problem:

$$
\begin{aligned}
& \text { minimize } \quad M S E_{\text {fit }}\left(\vec{x}_{f}\right) \\
& \vec{x}_{f} \\
& \text { subject to } \quad \vec{x}_{\min } \preccurlyeq_{\mathbb{R}_{+}^{5}} \vec{x}_{f} \preccurlyeq_{\mathbb{R}_{+}^{5}} \vec{x}_{\max }
\end{aligned}
$$

Where $M S E_{f i t}\left(\vec{x}_{f}\right)$ stands for the regression model, obtained in section $5.3 . \preccurlyeq_{\mathbb{R}_{+}^{5}}$ denotes the generalized inequality defined by the non negative $\mathbb{R}^{5}$ orthant $\left(\mathbb{R}_{+}^{5}\right)$. This generalized inequality corresponds to the componentwise vector inequality. The bond restrictions are included in the optimization problem since the model is only valid in the studied parameter range.

Due to the regression model form, this optimization problem is a quadratic optimization problem [34]. The convexity of the problem determines how difficult to solve is the optimization problem, and the appropriate resolution method [35]. The convexity of a quadratic optimization problem is given by the properties of the second order term matrix, $\boldsymbol{A}$ [36]. In this case, matrix $\boldsymbol{A}$ is an indefinite matrix: therefore, optimization problem (13) is a non-convex optimization problem. Such kind of problems is NP-hard [37]. There are algorithms for obtaining the global optimum of non convex quadratic optimization problems, such as the LPCC method [38] and the globally solving method based on completely positive programming [39]. However, in general, non linear programming methods are used to solve this kind of optimization problem: the most common ones being the active set method, the interior point method and the trust region method [40]. These algorithms are implemented in the quadprog function of the Mathlab® optimization toolbox [41]. Consequently, the aforementioned toolbox was used to solve the optimization problem in this work. The obtained optimum values are given in table 5 .

\subsection{EIS measurements with the optimum measurement parameters}

Figure 7 compares one of the 150 spectra obtained with NOVA®'s default measurement parameters used for the determination of the reference spectrum (the 
other 149 spectra present a similar pattern to the shown one); with the spectrum obtained with the optimum measurement parameters. The difference is significant: while the spectrum obtained with the optimum measurement parameters is nearly the reference spectrum; the spectrum obtained with NOVA®'s default measurement parameters presents a significant deviation from the reference spectrum. The spectrum obtained with the optimum measurements parameters gives the equivalent information to 150 spectra obtained with the default measurement parameters (since 150 default spectra were used to determine the reference spectrum).

The general practice in EIS is to fit the measured spectra to an equivalent electrical circuit [28]. For this reason, in order to quantify the difference between both spectra shown in figure 7, they were fitted to the reference equivalent circuit given in figure 1. The considered equivalent circuit consists in a $R(R C P E)$ circuit. It considers 4 parameters: the series resistance $\left(R_{S}\right)$, the parallel resistance $\left(R_{p}\right)$, and the constant phase element $\left(Q_{0}, \alpha\right)$. Table 6 shows the model parameters obtained from fitting the default parameter and the optimum parameter spectra. In the same table, the reference value of each parameter is given. Using these reference values, the relative error of each parameter, $\varepsilon_{i}$, was determined using the following expression:

$$
\varepsilon_{i}=\frac{\left|X_{i}-X_{i}(r e f)\right|}{X_{i}(r e f)}
$$

Where $X_{i}$ (ref) stands for the reference value for the $i$-th model parameter; and $X_{i}$ denotes the value of the corresponding parameter. Table 6 lists the relative errors for the model parameters determined from the fitting of the default and the optimum spectra. It can be observed that the relative errors of the parameters obtained from the default spectrum are more than 3 times bigger than the relative errors of the parameters obtained from the optimum spectrum, for every model parameter. The total relative error in the default case is $74 \%$, whereas in the optimum case it is only of $16 \%$ : the optimum measurement parameters lead to a total relative error with respect to the reference case 4.5 times lower than the default measurement parameters. This shows that there is a significant quantitative improvement of the EIS analysis results if the optimum measurement parameters are used for the EIS measurements.

Therefore, if the usual proceeding of not optimizing the measurement parameters and just taking the default values was done with the system studied in this work, the obtained spectra would have a significant error. This shows the importance of properly selecting the measurement parameters in order to get the best quality spectra that lead to model parameters with less error. 


\section{Conclusions}

In conclusion, the optimum impedance measurement parameters for the system studied in this work are: 1 second integration time and 5 integration cycles; 15 stabilization cycles, 1 second maximum stabilization time and a minimal fraction of 0 . These parameters lead to a spectrum of much higher quality (less error with respect to the reference spectrum) than the spectrum obtained with NOVA®'s default parameters. Therefore, the developed methodology allowed obtaining the optimal measurement parameters that minimized the spectrum error: the total relative error in the fitted model parameters was 4.5 times lower when the optimum measurement parameters were used instead of the default measurement parameters.

Additionally, this work highlights the importance of a proper selection of the impedance measurement parameters. Authors recommend that optimization of the measurement parameters is performed for each particular system, instead of the general practice of taking the default measurement parameter values.

The methodology presented in this work is quite powerful. However it has two major drawbacks: the reference spectrum must be known; and the optimum parameters may vary from an operation point to another. Regarding the first issue, the reference spectrum can always be determined (for every system and every operation condition) using the inferential methodology to obtain an accurate estimation of the "real" spectrum from a large number of measurements with the default measurement parameters. Regarding the second issue, in general the optimum measurement parameters can vary from one operation point to another. Consequently, the optimization should be done for every operation point. But this is not possible from a practical point of view. A compromise solution should be considered. The methodology presented in this work should be applied for average operation conditions. Rather than repeating the optimization for all the other operation conditions; the optimum measurement parameters for the average operation conditions are used for all the operation conditions. This may lead to suboptimal measurement parameters; but those measurement parameters will be closer to the optimum ones for each operation condition than the default ones. In a certain sense, the first optimization for the average operation conditions is used to determine the order of magnitude of the optimum measurement parameters for the studied system. Further work should be done in order to determine the variation of the optimum measurement conditions with the operation conditions; and therefore, the practical viability of the proposed compromise solution. 


\section{Nomenclature}

\section{$\underline{\text { Latin letters }}$}

$\boldsymbol{A} \quad$ Matrix of second order effects

$\vec{b} \quad$ Simple effect vector

$f \quad$ Frequency $(\mathrm{Hz})$

MSE Mean square error $\left(\Omega^{2}\right)$

$N_{f} \quad$ Number of frequencies at which the impedance is measured

$t_{a}(f) \quad$ Duration of the acquisition step for frequency $f(s)$

$t_{m}(f) \quad$ Measurement time for frequency $f(s)$

$t_{\text {tot }} \quad$ Total measurement time $(s)$

$t_{s}(f) \quad$ Duration of the stabilization step for frequency $f(s)$

$\vec{x}_{f} \quad$ Vector of factors

$Z \quad$ Complex electrochemical impedance $(\Omega)$

$Z^{\prime} \quad$ Real part of the electrochemical impedance $(\Omega)$

$Z^{\prime \prime} \quad$ Imaginary part of the electrochemical impedance $(\Omega)$

\section{Greek letters}

$\begin{array}{ll}\varepsilon_{i} & \text { Relative error } \\ \xi_{S S} & \text { Minimum stabilization cycle fraction } \\ \tau_{I} & \text { Maximum integration time }(s) \\ \tau_{S S} & \text { Maximum stabilization time }(s) \\ v_{I} & \text { Minimum number of integration cycles } \\ v_{S S} & \text { Number of stabilization cycles } \\ \omega & \text { Angular frequency }\left(\mathrm{rad} \cdot \mathrm{s}^{-1}\right)\end{array}$

\section{Subscripts}

fit $\quad$ Fitting of the experimental spectrum

ref Reference spectrum 


\section{Acknowledgments}

The authors are very grateful to the Generalitat Valenciana for its economic support in form of Vali+d grant (Ref: ACIF-2013-268). 


\section{References}

[1] N. Wagner, Electrochemical impedance spectroscopy, in: H. Wang, X.Z. Yuan, PEM Fuel Cell Durability Handbook, CRC Press, New York, 2011, p. 37.

[2] O. Reid, F.S. Saleh, E.B. Easton, Determining electrochemically active surface area in PEM fuel cell electrodes with electrochemical impedance spectroscopy and its application to catalyst durability, Electrochim. Acta 114 (2013) 278.

[3] N. Wagner, K.A. Friedrich, Application of electrochemical impedance spectroscopy for fuel cell characterization: PEFC and oxygen reduction reaction in alkaline solution, Fuel Cells 9 (2009) 237.

[4] M. Mamlouk, K. Scott, Analysis of high temperature polymer electrolyte membrane fuel cell electrodes using electrochemical impedance spectroscopy, Electrochim. Acta 56 (2011) 5493.

[5] C. Boyer, S. Gamburzev, O. Velev, S. Srinivasan, A.J. Appleby, Measurement of proton conductivity in the active layer of PEM fuel cell gas diffusion electrodes, Electrochim. Acta 43 (1998) 3703.

[6] C. Zhang, L. Zhang, W. Zhou, Y. Wang, S.W. Chan, Investigation of water transport and its effects on performance of high-temperature PEM fuel cells, Electrochim. Acta 149 (2014) 271.

[7] H. Nara, T. Momma, T. Osaka, Impedance analysis of the effect of flooding in the cathode catalyst layer of the polymer electrolyte fuel cell, Electrochim. Acta 113 (2013) 720.

[8] N. Wagner, T. Kaz, K.A. Friedrich, Investigation of electrode composition of polymer fuel cells by electrochemical impedance spectroscopy, Electrochim. Acta 53 (2008) 7475.

[9] H. Tang, M. Pan, S.P. Jiang, X. Wang, Y. Ruan, Fabrication and characterization of PFSI/ePTFE composite proton exchange membranes of polymer electrolyte fuel cells, Electrochim. Acta 52 (2007) 5304.

[10] P. Gode, F. Jaouen, G. Lindbergh, A. Lundblad, G. Sundholm, Influence of the composition on the structure and electrochemical characteristics of the PEFC cathode, Electrochim. Acta 48 (2003) 4175. 
[11] S. Uhm, Y. Kwon, S.T. Chung, J. Lee, Highly effective anode structure in a direct formic acid fuel cell, Electrochim. Acta 53 (2008) 5162.

[12] R. Padmavathi, R. Karthikumar, D. Sangeetha, Multilayered sulphonated polysulfone/silica composite membranes for fuel cell applications, Electrochim. Acta 71 (2012) 283.

[13] F. Liu, C.Y. Wang, Optimization of cathode catalyst layer for direct metanol fuel cells: Part I. Experimental investigation, Electrochim. Acta 52 (2006) 1417.

[14] Y. Tabuchi, T. Shiomi, O. Aoki, N. Kubo, K. Shinohara, Effects of heat and water transport on the performance of polymer electrolyte membrane fuel cell under high current density operation, Electrochim. Acta 56 (2010) 352.

[15] Y.H. Cho, S.J. Yoo, I.S. Park, T.Y. Jeon, Y.H. Cho, J.W Lim, O.J. Kwon, W.S. Yoon, Y.E Sung, Characteristics and performance of membrane electrode assemblies with operating conditions in polymer electrolyte membrane fuel cell, Electrochim. Acta 56 (2010) 717.

[16] M. Schulze, N. Wagner, T. Kaz, K.A. Friedrich, Combined electrochemical and surface analysis investigation of degradation processes in polymer electrolyte membrane fuel cells, Electrochim. Acta 52 (2007) 2328.

[17] D. Yang, J. Ma, L. Xu, M. Wu, H. Wang, The effect of nitrogen oxides in air on the performance of proton exchange membrane fuel cell, Electrochim. Acta 51 (2006) 4039.

[18] A. Hakenjos, M. Zobel, J. Clausnitzer, C. Habling, Simultaneous electrochemical impedance spectroscopy of single cells in a PEM fuel cell stack, J of Power Sources 154 (2006) 360.

[19] S. Tant, S. Rosini, P.X. Thivel, F. Druart, A. Rakotondrainibe, T. Geneston, Y. Bultel, An algorithm for diagnosis of proton exchange membrane fuel cells by electrochemical impedance spectroscopy, Electrochim. Acta 135 (2014) 368.

[20] C. Comminges, Q.X. Fu, M. Zahidi, N.Y. Steiner, O. Bucheli, Monitoring the degradation of a solid oxide fuel cell stach during $10000 \mathrm{~h}$ via electrochemical impedance spectroscopy, Electrochim. Acta 59 (2012) 367. 
[21] H. Xu, H.R. Kunz, J.M. Fenton, Analysis of proton exchange fuel cell polarization losses at elevated temperature $120^{\circ} \mathrm{C}$ and reduced relative humidity, Electrochim. Acta 52 (2007) 3525.

[22] S. Takaichi, H. Uchida, M. Watanabe, In situ analysis of oxygen partial pressure at the cathode catalyst layer/membrane interface during PEFC operation, Electrochim. Acta 53 (2008) 4699.

[23] K. Wikander, H. Ekström, A.E.C Palmqvist, G. Lindbergh, On the influence of Pt particle size on the PEMFC cathode performance, Electrochim. Acta 52 (2007) 6848.

[24] J.C. Amphlett, B.A. Peppley, E. Halliop, A. Sadiq, The effect of anode flow characteristics and temperature on the performance of a direct methanol fuel cell, $\mathrm{J}$ of Power Sources 96 (2001) 204.

[25] C. Lamy, D.J. Jones, C. Coutanceau, P. Brault, S. Martemianov, Y. Bultel, Do not forget the electrochemical characteristics of the membrane electrode assembly when designing a Proton Exchange Membrane fuel cell stack, Electrochim. Acta 56 (2011) 10406.

[26] E. Barsoukov, J.R. Macdonald, Impedance spectroscopy. Theory, experiment and applications, 2nd ed., John Wiley \& Sons, New York, 2005.

[27] X.Z. Yuan, C. Song, H. Wang, J. Zhang, Electrochemical impedance spectroscopy in PEM fuel cells. Fundamentals and applications, Springer, London, 2010.

[28] M.E. Orazem, B. Tribollet, Electrochemical impedance spectroscopy, John Wiley \& Sons, New Jersey, 2008.

[29] Metrohm Autolab. NOVA tutorial. Utrecht; 2012.

[30] D.C. Montgomery, Design and analysis of experiments, 7th ed., John Wiley \& Sons, London, 2009.

[31] G. Box, S. Hunter, W. Hunter, Statistics for experimenters. Design, innovation, and discovery, Wiley-Interscience, Hoboken, 2005.

[32] W. Mendenhall, T. Sincich, Statistics for engineering and the sciences, 5th ed., Pearson Education, London, 2007.

[33] F. Barbir, PEM fuel cells, Elsevier Academic Press, London, 2005. 
[34] S. Boyd, L. Vandenberghe, Convex optimization, Cambridge University Press, Cambridge, 2004.

[35] M.A. Hanson, Nonconvex quadratic programming. Department of Statistics, Tallahassee, FSU technical report M-810; 1990.

[36] J. Nocedal, S. Wright, Numerical optimization, 2nd ed, Springer, New York, 2006.

[37] P.M. Pardalos, S.A. Vavasis, Quadratic programming with one negative eigenvalue is NP-hard, Global optimization 1 (1992) 87.

[38] J. Hu, J.E. Mitchell, J.S Pang, An LPCC approach to nonconvex quadratic programs, Math. Program. J. 133 (2012) 243.

[39] J. Chen, S. Burer, Globally solving nonconvex quadratic programming problems via completely positive programming, Math. Program. J. 4 (2012) 33.

[40] N.I.M Gould, P.L. Toint, Numerical methods for large-scale non-convex quadratic programming, Appl. Math. Opt. 72 (2002) 149.

[41] The MathWorks Inc. MathWorks: MATLAB reference guide. Natick; 2010. 


\section{LIST OF TABLES.}

Table 1. Quantitative values of the encoded factor levels

Table 2. ANOVA table for $\boldsymbol{M S} \boldsymbol{E}_{\text {fit }}$ parameter

Table 3. Regresion model coefficients

Table 4. ANOVA table for the model significance test

Table 5. Optimum value for each one of the measurement parameters

Table 6. Comparison of the model parameters obtained using default and optimum 


\section{LIST OF FIGURES.}

Figure 1. Reference spectrum and electric equivalent circuit

Figure 2. Outline of the methodology used in this work

Figure 3. Experimental setup

Figure 4. Results for experiment $(-;-;-;-;-)$ (Integration time: $0.1 \mathrm{~s}$; Number of integration cycles: 1 cycle; Number of stabilization cycles: 5 cycles; Stabilization time: 1 s; Minimum cycle fraction: 0 )

Figure 5. Results for experiment $(+;+;-;-;+)$ (Integration time: $1 \mathrm{~s}$; Number of integration cycles: 5 cycles; Number of stabilization cycles: 5 cycles; Stabilization time: $1 \mathrm{~s}$; Minimum cycle fraction: 0.5 )

Figure 6. Pareto chart for the mean square error of the experimental data fit with respect to the reference data, built for a confidence level of $95.4 \%$

Figure 7. Spectra measured with the optimum measurement parameters and with the NOVA® default parameters 
Table 1. Quantitative values of the encoded factor levels

\begin{tabular}{|c|c|c|c|c|}
\hline Factor & Level - 1 & Level 0 & Level + 1 & $\begin{array}{l}\text { Software } \\
\text { default }\end{array}$ \\
\hline$\tau_{I}(s)$ & 0.10 & 0.55 & 1.00 & 0.125 \\
\hline$v_{I}($ Cycles $)$ & 1 & 3 & 5 & 1 \\
\hline$v_{S S}($ Cycles $)$ & 5 & 10 & 15 & 10 \\
\hline$\tau_{S S}(s)$ & 1.0 & 20.5 & 40.0 & 1.0 \\
\hline$\xi_{s s}$ & 0.00 & 0.25 & 0.50 & 0.00 \\
\hline
\end{tabular}


Table 2. ANOVA table for $\boldsymbol{M S} \boldsymbol{E}_{\text {fit }}$ parameter

\begin{tabular}{|c|c|c|c|c|c|}
\hline Factor & $\begin{array}{l}\text { Sum of } \\
\text { squares }\end{array}$ & $\begin{array}{l}\text { Degrees of } \\
\text { freedom }\end{array}$ & $\begin{array}{l}\text { Mean } \\
\text { square }\end{array}$ & F ratio & p-value \\
\hline$\tau_{I}$ & $3.30 \cdot 10^{-11}$ & 1 & $3.30 \cdot 10^{-11}$ & 3.05 & 0.0986 \\
\hline$v_{I}$ & $6.51 \cdot 10^{-11}$ & 1 & $6.51 \cdot 10^{-11}$ & 6.03 & 0.0252 \\
\hline$v_{S S}$ & $7.49 \cdot 10^{-11}$ & 1 & $7.49 \cdot 10^{-11}$ & 6.93 & 0.0174 \\
\hline$\tau_{S S}$ & $1.15 \cdot 10^{-10}$ & 1 & $1.15 \cdot 10^{-10}$ & 10.64 & 0.0046 \\
\hline$\xi_{s S}$ & $6.37 \cdot 10^{-12}$ & 1 & $6.37 \cdot 10^{-12}$ & 0.59 & 0.4531 \\
\hline$\tau_{I} \cdot v_{I}$ & $6.40 \cdot 10^{-11}$ & 1 & $6.40 \cdot 10^{-11}$ & 5.92 & 0.0263 \\
\hline$\tau_{I} \cdot v_{S S}$ & $3.11 \cdot 10^{-13}$ & 1 & $3.11 \cdot 10^{-13}$ & 0.03 & 0.8673 \\
\hline$\tau_{I} \cdot \tau_{S S}$ & $1.04 \cdot 10^{-11}$ & 1 & $1.04 \cdot 10^{-11}$ & 0.96 & 0.3414 \\
\hline$\tau_{I} \cdot \xi_{S S}$ & $2.50 \cdot 10^{-11}$ & 1 & $2.50 \cdot 10^{-11}$ & 2.31 & 0.1468 \\
\hline$v_{I} \cdot v_{S S}$ & $2.91 \cdot 10^{-13}$ & 1 & $2.91 \cdot 10^{-13}$ & 0.03 & 0.8715 \\
\hline$v_{I} \cdot \tau_{S S}$ & $2.21 \cdot 10^{-11}$ & 1 & $2.21 \cdot 10^{-11}$ & 2.04 & 0.1709 \\
\hline$v_{I} \cdot \xi_{s S}$ & $1.04 \cdot 10^{-12}$ & 1 & $1.04 \cdot 10^{-12}$ & 0.10 & 0.7599 \\
\hline$v_{S S} \cdot \tau_{S S}$ & $9.62 \cdot 10^{-12}$ & 1 & $9.62 \cdot 10^{-12}$ & 0.89 & 0.3585 \\
\hline$v_{S S} \cdot \xi_{S S}$ & $1.11 \cdot 10^{-13}$ & 1 & $1.11 \cdot 10^{-13}$ & 0.01 & 0.9205 \\
\hline$\tau_{S S} \cdot \xi_{S S}$ & $7.85 \cdot 10^{-11}$ & 1 & $7.85 \cdot 10^{-11}$ & 7.27 & 0.0153 \\
\hline Residual & $1.84 \cdot 10^{-10}$ & 17 & $1.08 \cdot 10^{-11}$ & & \\
\hline Total & $6.89 \cdot 10^{-10}$ & 32 & & & \\
\hline
\end{tabular}


Table 3. Regresion model coefficients

\begin{tabular}{|c|c|c|}
\hline $\begin{array}{c}\text { Model } \\
\text { parameter }\end{array}$ & $\begin{array}{c}\text { Estimated } \\
\text { value }\end{array}$ & Units \\
\hline$c$ & $8.84 \cdot 10^{-6}$ & $\Omega^{2}$ \\
\hline$b_{\tau_{I}}$ & $-6.97 \cdot 10^{-6}$ & $\Omega^{2} \cdot s^{-1}$ \\
\hline$b_{v_{I}}$ & $-1.58 \cdot 10^{-6}$ & $\Omega^{2}$ \\
\hline$b_{v_{S S}}$ & $-3.06 \cdot 10^{-7}$ & $\Omega^{2}$ \\
\hline$b_{\tau_{S S}}$ & $1.69 \cdot 10^{-8}$ & $\Omega^{2} \cdot s^{-1}$ \\
\hline$b_{\xi_{S S}}$ & $8.37 \cdot 10^{-6}$ & $\Omega^{2}$ \\
\hline$a_{\tau_{I} \cdot v_{I}}$ & $-1.57 \cdot 10^{-6}$ & $\Omega^{2} \cdot s^{-1}$ \\
\hline$a_{\tau_{S S} \cdot \xi_{S S}}$ & $3.21 \cdot 10^{-7}$ & $\Omega^{2} \cdot s^{-1}$ \\
\hline$R^{2}$ & $83.35 \%$ & \\
\hline
\end{tabular}


Table 4. ANOVA table for the model significance test

\begin{tabular}{|c|c|c|c|c|c|}
\hline Factor & $\begin{array}{l}\text { Sum of } \\
\text { squares }\end{array}$ & $\begin{array}{l}\text { Degrees of } \\
\text { freedom }\end{array}$ & $\begin{array}{c}\text { Mean } \\
\text { square }\end{array}$ & F ratio & p-value \\
\hline Model & $2.94 \cdot 10^{-10}$ & 7 & $4.20 \cdot 10^{-11}$ & 2.66 & 0.0104 \\
\hline Residual & $3.95 \cdot 10^{-10}$ & 25 & $1.58 \cdot 10^{-11}$ & & \\
\hline Total & $6.89 \cdot 10^{-10}$ & 32 & & & \\
\hline
\end{tabular}


Table 5. Optimum value for each one of the measurement parameters Measurement Optimum parameter value

\begin{tabular}{|c|c|}
\hline $\boldsymbol{\tau}_{\boldsymbol{I}}$ & $1.0 \mathrm{~s}$ \\
\hline $\boldsymbol{v}_{\boldsymbol{I}}$ & 5 cycles \\
\hline $\boldsymbol{v}_{\boldsymbol{S S}}$ & 15 cycles \\
\hline $\boldsymbol{\tau}_{\boldsymbol{S S}}$ & $1.0 \mathrm{~s}$ \\
\hline $\boldsymbol{\xi}_{\boldsymbol{S S}}$ & 0.00 \\
\hline
\end{tabular}


Table 6. Comparison of the model parameters obtained using default and optimum measurement parameters

\begin{tabular}{|c|c|c|c|c|c|}
\hline \multirow{2}{*}{ Model parameter } & \multirow{2}{*}{ Reference } & \multicolumn{2}{|c|}{ Default } & \multicolumn{2}{c|}{ Optimum } \\
\cline { 3 - 6 } & & Value & Relative error (\%) & Value & Relative error (\%) \\
\hline $\boldsymbol{R}_{\boldsymbol{S}}(\boldsymbol{\Omega})$ & 0.00573 & 0.00612 & 7.17 & 0.00575 & 0.69 \\
\hline $\boldsymbol{R}_{\boldsymbol{p}}(\boldsymbol{\Omega})$ & 0.0383 & 0.0413 & 7.58 & 0.0386 & 0.71 \\
\hline $\boldsymbol{Q}_{\mathbf{0}}\left(\boldsymbol{F} \cdot \boldsymbol{s}^{\boldsymbol{\alpha - 1}}\right)$ & 0.4391 & 0.2248 & 48.81 & 0.3773 & 14.06 \\
\hline $\boldsymbol{\alpha}$ & 0.8928 & 0.9890 & 10.78 & 0.8860 & 0.77 \\
\hline \multicolumn{2}{|c}{} & Total & 74.33 & Total & 16.23 \\
\cline { 3 - 6 }
\end{tabular}




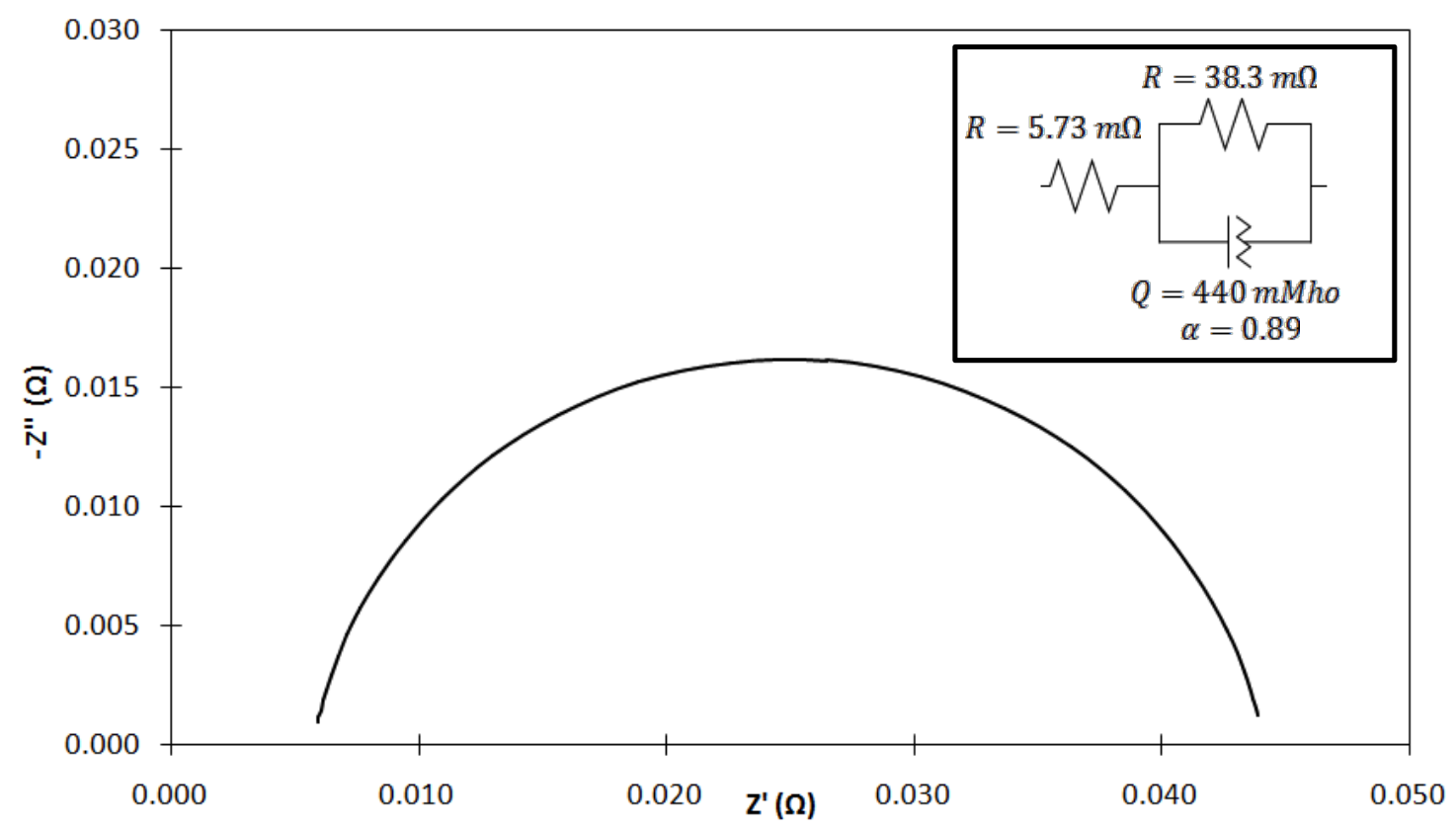

Figure 1. Reference spectrum and electric equivalent circuit 


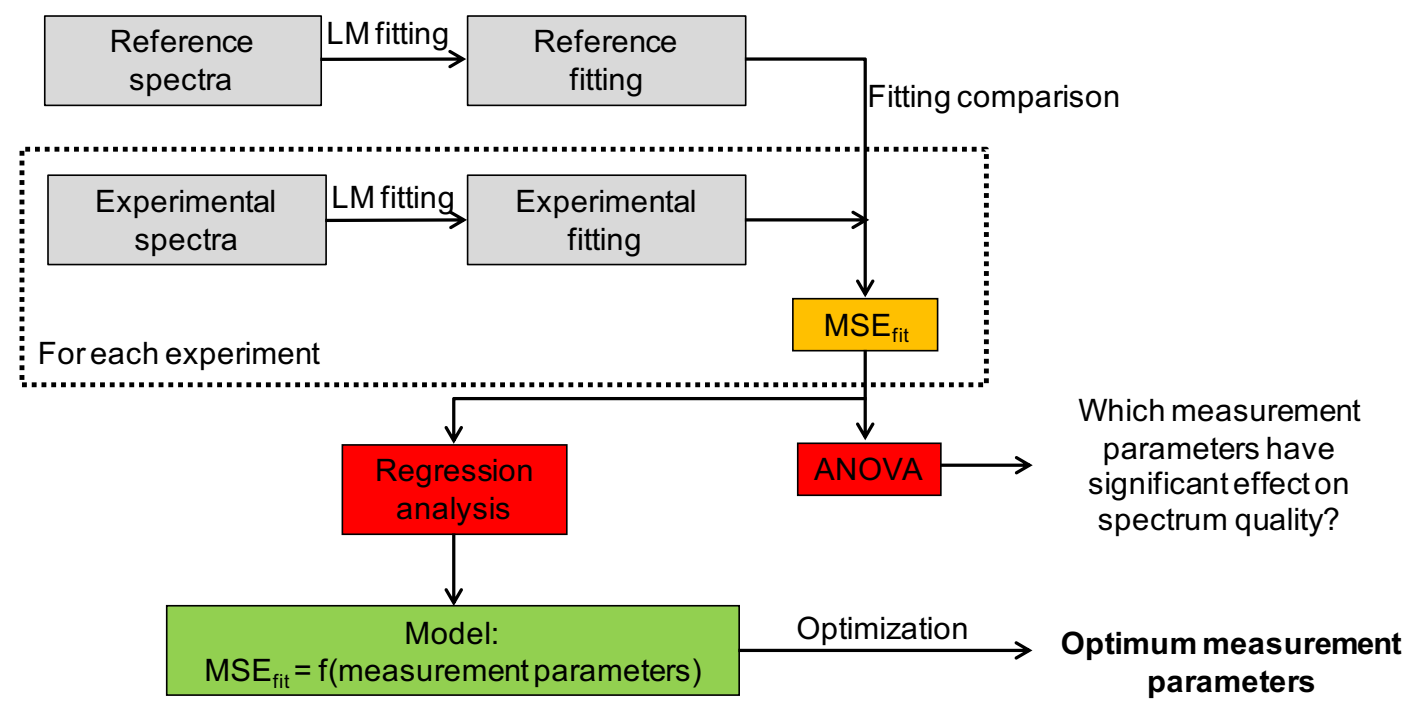

Figure 2. Outline of the methodology used in this work 


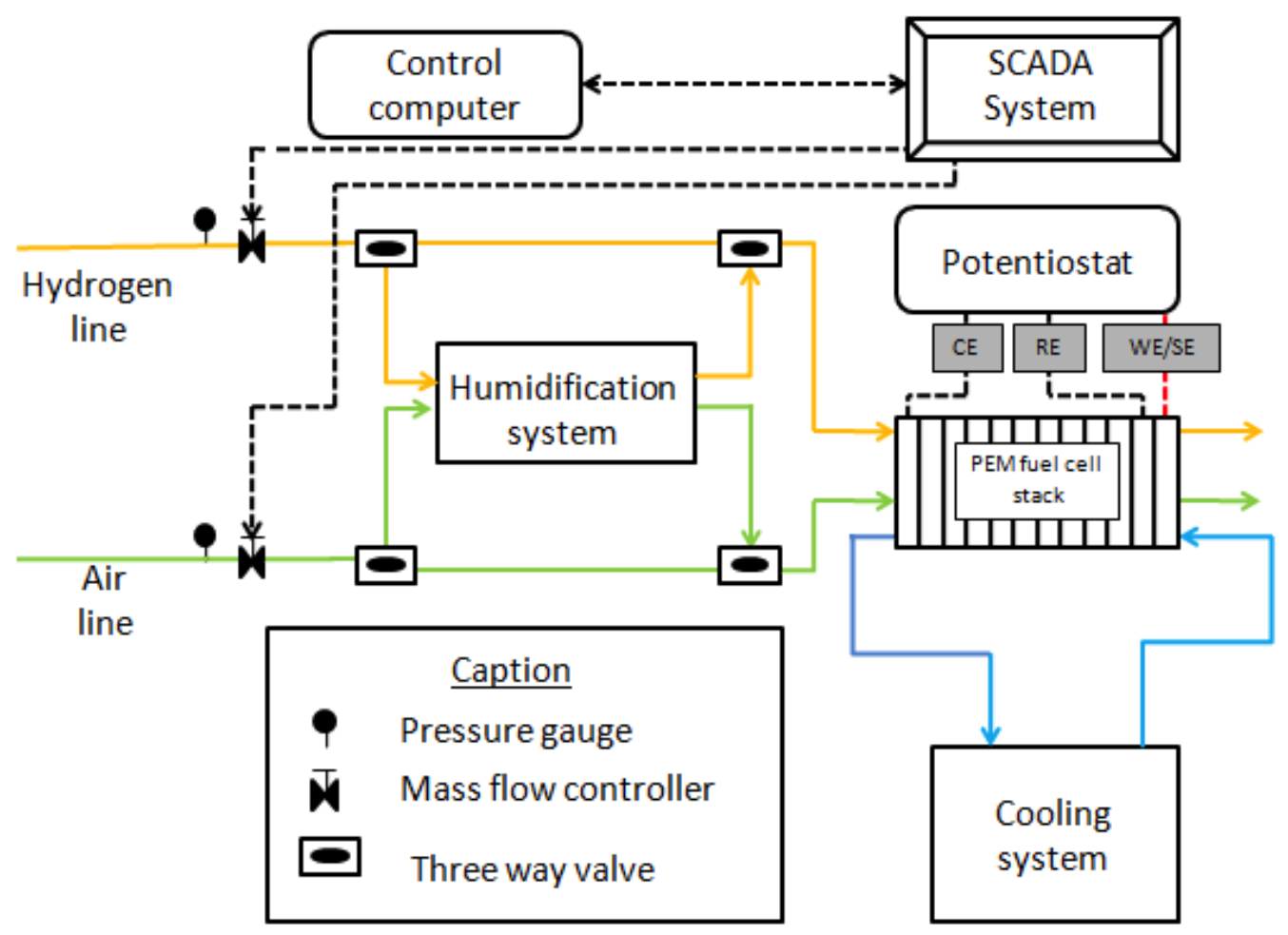

Figure 3. Experimental setup 


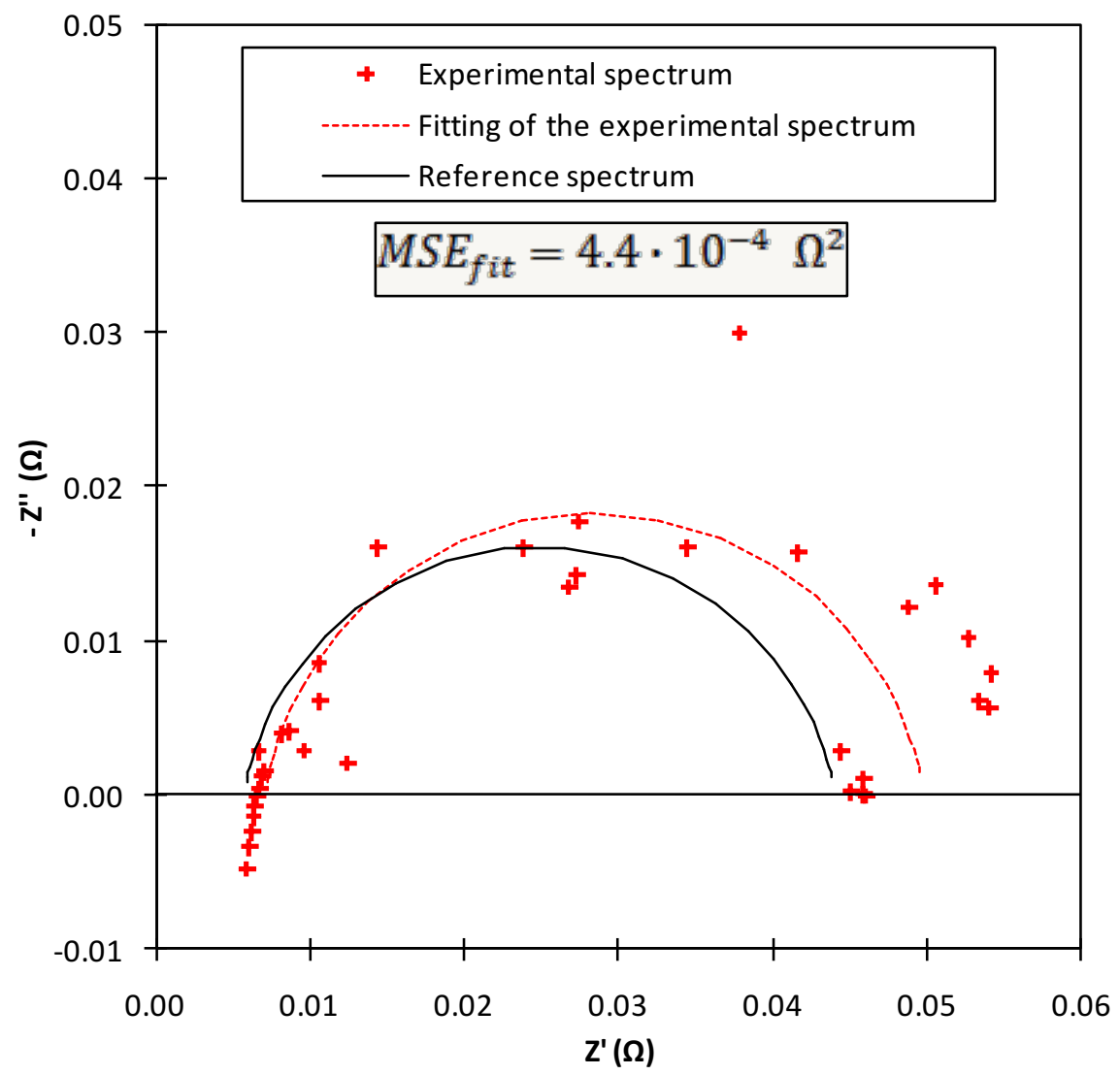

Figure 4. Results for experiment (-; -; -; -; -) (Integration time: $0.1 \mathrm{~s}$; Number of integration cycles: 1 cycle; Number of stabilization cycles: 5 cycles; Stabilization time: 1 s; Minimum cycle fraction: 0 ) 


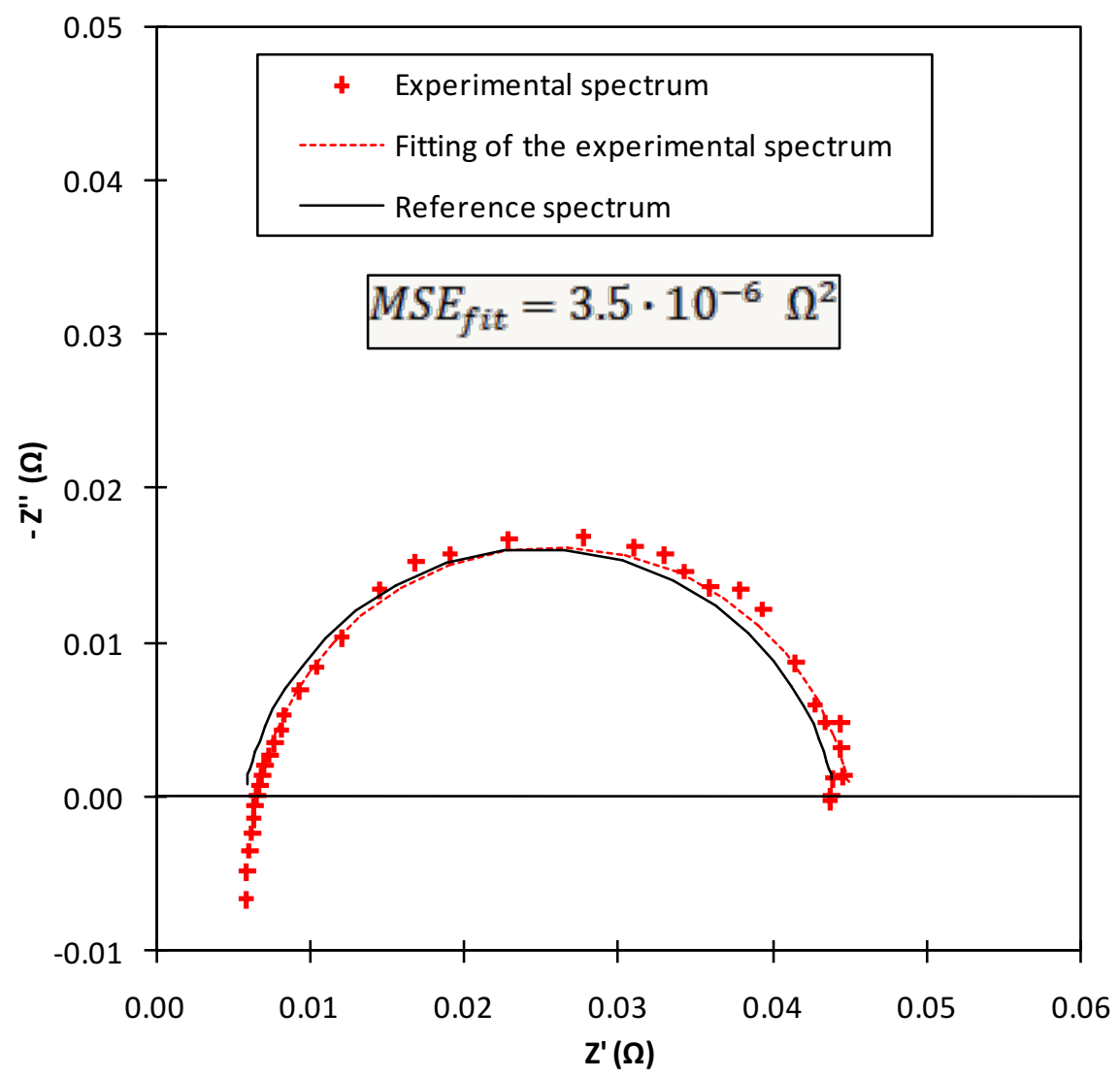

Figure 5. Results for experiment $(+;+;-;-;+)$ (Integration time: $1 \mathrm{~s}$; Number of integration cycles: 5 cycles; Number of stabilization cycles: 5 cycles; Stabilization time: $1 \mathrm{~s}$; Minimum cycle fraction: 0.5 ) 


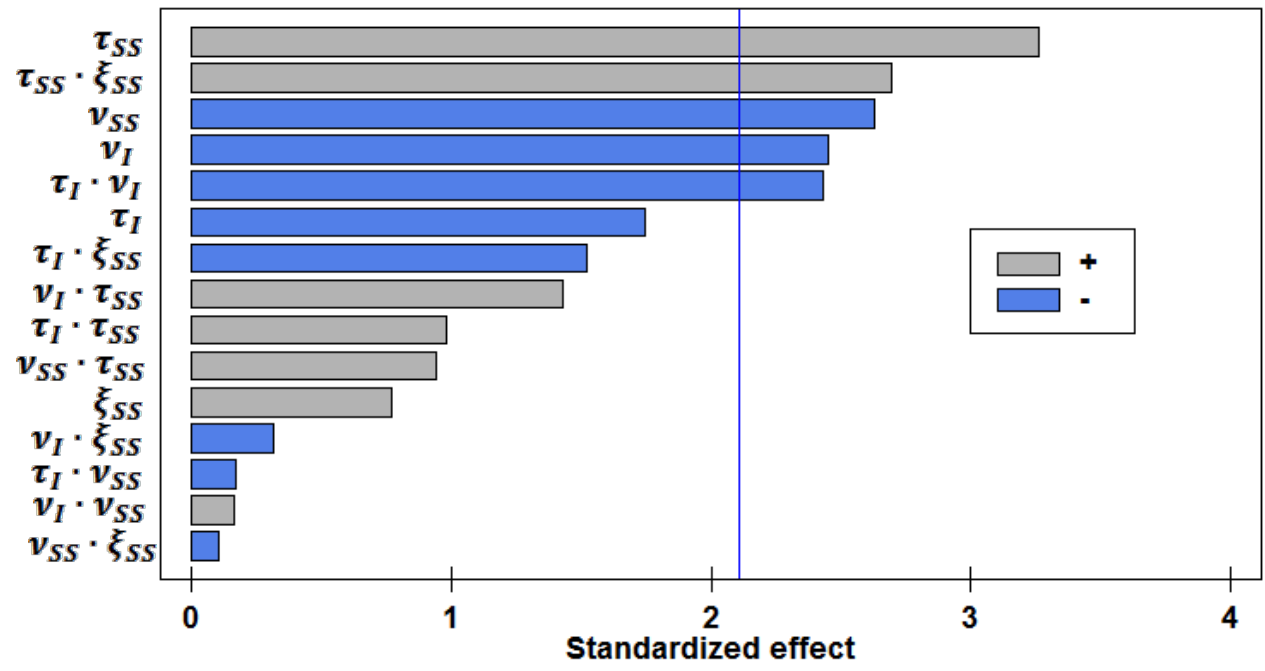

Figure 6. Pareto chart for the mean square error of the experimental data fit with respect to the reference data, built for a confidence level of $95.4 \%$ 


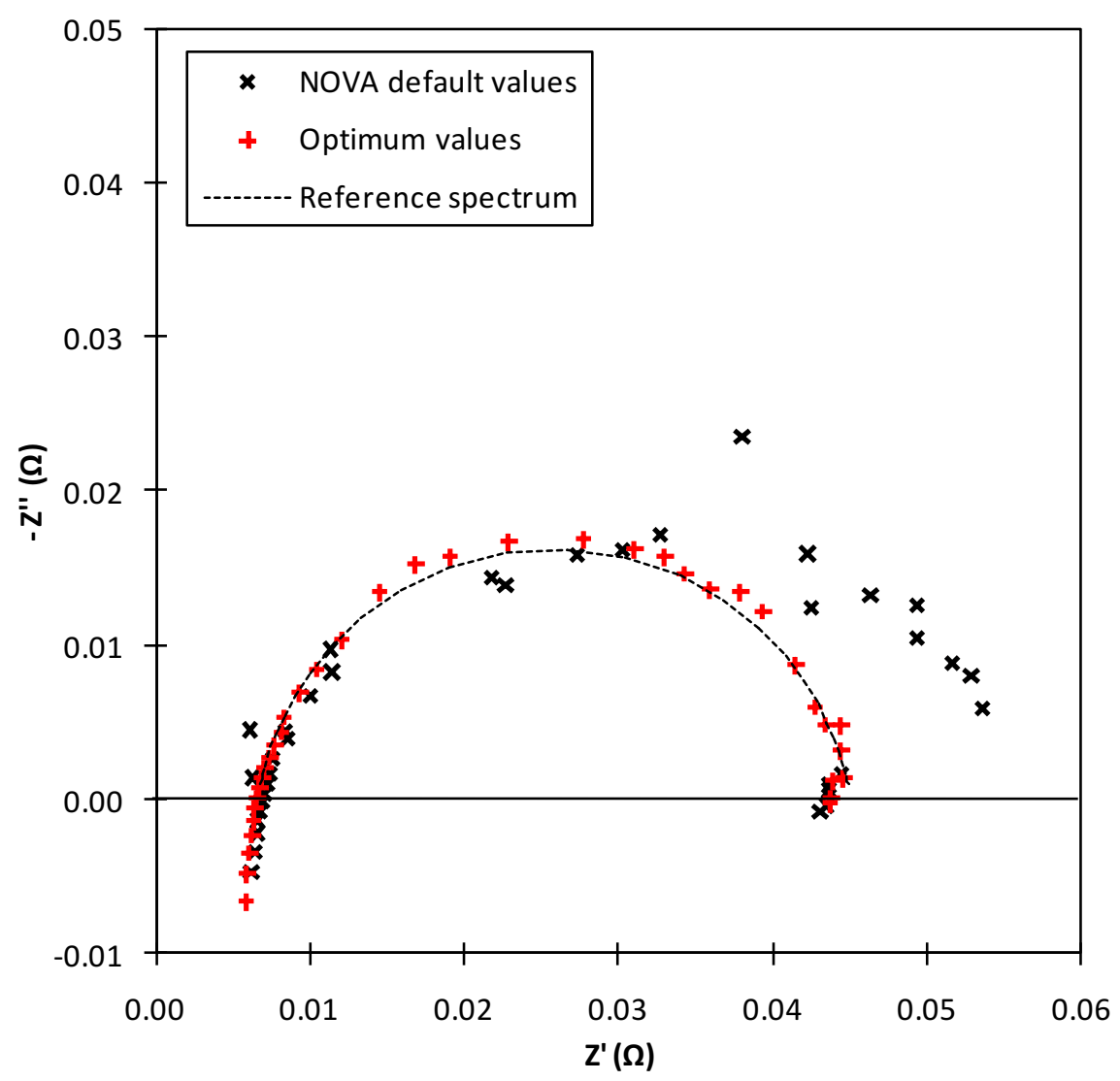

Figure 7. Spectra measured with the optimum measurement parameters and with the NOVA® default parameters 